\title{
MAIN PANCREATIC DUCT (MPD) DIAMETER: CONTRAST ENHANCED COMPUTED TOMOGRAPHY (CECT) MEASUREMENT IN NORMAL SUBJECTS IN SRI LANKA.
}

\author{
Wijayapala M H W M N ${ }^{1}$, Ranasinghe D D ${ }^{2}$ \\ ${ }^{1}$ Department of Radiology, NHSL,Colombo, Sri Lanka \\ ${ }^{2}$ Department of Radiology, CSTH, Kalubowila, Sri Lanka
}

Keywords : Pancreatic duct; Computed tomography; pancreatic cancer; pancreatitis

Corresponding Author: Wijayapala M H W M N ${ }^{1}$

Copyright: Wijayapala M H W M N ${ }^{1}$

iD https://orcid.org/0000-0002-5984-5118

\section{Introduction}

Dilatation of the MPD is associated with number of significant pancreatic pathologies. To identify the dilated MPD, it is of great importance to study the demographic data of the normal size of the MPD in a given population.

\section{Objective}

To assess the diameter of MPD in normal subjects.

\section{Methodology}

Prospective descriptive study was carried out at Colombo South Teaching Hospital from March 2016 to May 2016. All CECT abdomen and pelvis examinations performed during this period with clinical, biochemical and radiological evidence of normal pancreas were included. CT measurements of the MPD diameter were obtained by two individuals independently on the subjects who underwent abdominal CECT scans (16 section MDCT, 1-5mm increments, and dual phase) and each measurement was obtained in venous phase images for three times and mean value was taken.

\section{Results}

There were 190 men and 132 women with age ranging from 20-88 years (mean 53.6). MPD was adequately visualized only in $72 \%(\mathrm{n}=231)$ of subjects. The mean diameter of the MPD in the head, body and tail were 2.9, 2.2 and $1.6 \mathrm{~mm}$ respectively. There is no statistical significant difference observed in MPD diameter between two sexes and the two age groups of $<40$ and $>40$ years $(p>0.05)$. 


\section{Conclusion}

The diameter values of MPD in our study are comparable to that of foreign population groups. However there was no correlation between MPD diameter with the age or sex in the study which was different to the published data which showed higher proportion of MPD diameter with increasing age and female gender.

\section{Introduction}

\section{Background and justification}

Dilatation of the MPD is associated with number of significant pancreatic pathologies such as acute/chronic pancreatitis, pancreatic malignancy and intraductal papillary mucinous neoplasms. To identify the dilated MPD, it is of great importance to study the demographic data of the normal size of the MPD in a given population.

According to available studies, the normal diameter of MPD in a healthy individual is somewhat dissimilar in different parts of the pancreas ${ }^{1}$. However, the MPD diameter is comparable in studied population groups, for example in Asia, Europe and America ${ }^{1}$. While numerous prior studies have published the diameter of MPD in foreign populations, the MPD diameter in Sri Lankan population was seldom reported.

Since CT is commonly performed to identify the pancreatic pathologies, it is prudent to define the size of the normal MPD on CT to compare with the MPD associated with pancreatic pathologies.

"Pancreatic duct width measurements are now used as one of the major criteria for diagnosing certain pancreatic diseases." ${ }^{1}$ Some authors suggest "MPD dilatation $(\geq 2.5 \mathrm{~mm})$ without any obvious cause as an independent predictor of developing pancreatic cancer." 2 According to the results of Sartoi et al conducted in 2009, diameter of MPD is useful for prediction of malignancy ${ }^{3}$.

Thus, the purpose of our study was to assess the size of the MPD in normal subjects in a CT series in Sri Lanka. Age and sex related MPD diameter was also assessed. Normal Sri Lankan reference values of MPD diameter in cooperated with age and sex differences would be 
invaluable in early detection of pancreatic carcinoma and in the continued assessment of patients with pancreatitis and pancreatic cancer in the local population.

\section{Literature review}

According to a study of 233 ultrasound scans done on 49 normal individuals conducted by Hadidi A, "the mean diameter of the duct in the head-neck was $3 \mathrm{~mm}$; in the body proximal and distal to the neck, duct diameters were 2.1 and $1.6 \mathrm{~mm}$, respectively." 4

Study done by Mark D Edge et al on scientific implication of dilated main pancreatic duct on computed tomography says that "there are no strict criteria for diagnosis of MPD dilation, generally if the MPD measures greater than $3 \mathrm{~mm}$ in the head and $2 \mathrm{~mm}$ in the body or tail of the pancreas, it is considered enlarged." 1

Mortelé et al describes that "the duct diameter is greatest at the head and neck region and is slightly narrower towards the body and tail with normal reported value ranges between 1-3.5 mm."5

The diameter of the MPD is usually considered to enlarge with age, According to a retrospective study done in Japan showed that "the higher age group to have a higher proportion of MPD dilatation." Similar study by Glaser et al stated that "the age-dependent duct dilatation was significant $(\mathrm{P}<0.01)$ for persons who were 40 or more years old in comparison with people aged up to 39 years and distinct enlargement $(\mathrm{P}<0.01)$ of MPD was calculated in females, while males showed only a tendency towards dilatation."7

\section{Methods}

Subjects:

This descriptive study was conducted at the Radiology department of Colombo South Teaching Hospital. Patients who underwent contrast enhanced abdominal CT scans from March 2016 to May 2016 were taken as the study population. Study was conducted after obtaining approval from the Ethics review committee of the Colombo South Teaching Hospital.

Informed consents were obtained from all patients to analyse their clinical data in CT.

\section{Data collection:}

Initially patient demographic details and other relevant clinical information were collected by a registrar in Radiology. It included patient's sex, age, relevant clinical history and results of investigation (radiological, biochemical and histological). Patients with clinical or biochemical evidence of pancreatic disease, hepato-biliary disease, gall stones and diabetes were excluded from the study. Patients with CT evidence of pancreatic disease, hepato-biliary disease and gall stones were also omitted from the study. CT evaluation of the MPD was performed on the remaining subjects. Out of the above mentioned patients the diameter of the MPD was measured only when the pancreatic duct was adequately visualized on CT. 
Oral water and intravenous iodinated contrast (150 $\mathrm{mL}$ of Omnipaque) were used in all CTs. All patients were studied with 16-section multi detector computed tomography (MDCT), and images were obtained at $1-5 \mathrm{~mm}$ increments. CTs were performed with dual-phase acquisition. The CT evaluations were performed independently by two experienced registrars in Radiology under the supervision of Consultant Radiologists. The diameter of the MPD was measured in the venous phase images (70s post contrast) at the duct of Wirsung in the head, body and tail of the pancreas, between the outside border of the anterior and posterior walls of the duct. The diameter of the duct in the body of the pancreas was measured at mid body, just above the superior mesenteric artery. Every measurement was repeated three times and the data conveyed as an average value.

Confidentiality of the patients was maintained by not transferring the patient's name in to data collection sheet. (As only the BHT numbers were taken).

Statistics:

Patients' demographic data was statistically described in terms of frequencies and percentages. MPD diameter in different sexes and different age groups were expressed in mean values. Association between MPD diameter with the age and sex was analyzed using Chi- squared test. (Comparison of the categorical data) $\mathrm{P}$ value $<0.05$ was taken as statistically significant. SPSS 17.0 computer programme system was used for all statistical calculations.

\section{Results}

Participant's characteristics:

CT evaluations of pancreatic duct were carried out in 322 patients. There were $190(59 \%)$ men and $132(41 \%)$ women (Figure 1) with age ranging from 20-88 years (mean 53.6). (Figure 2) MPD was adequately visualized only in $72 \%(n=231)$ of subjects. (Figure 3$)$

Results of the MPD size measurements:

The mean diameter of the MPD in the head was $2.9 \pm 0.4 \mathrm{~mm}$. Minimum value obtained was $1.8 \mathrm{~mm}$ and maximum value obtained was $4.5 \mathrm{~mm}$. (Table 1)

The mean diameter of the MPD in the body was $2.2 \pm 0.3 \mathrm{~mm}$. Minimum value obtained was 1.5 $\mathrm{mm}$ and maximum value obtained was $3.4 \mathrm{~mm}$. (Table 1 )

The mean diameter of the MPD in the tail was $1.6 \pm 0.2 \mathrm{~mm}$. Minimum value obtained was 1.5 $\mathrm{mm}$ and maximum value obtained was $3.4 \mathrm{~mm}$. (Table 1 )

MPD diameter in males and females were assessed separately. In females MPD diameter in head, body and tail region were $2.9 \mathrm{~mm}, 2.2 \mathrm{~mm}, 1.6 \mathrm{~mm}$ respectively. In males MPD diameter in head, body and tail region were $2.9 \mathrm{~mm}, 2.2 \mathrm{~mm}, 1.6 \mathrm{~mm}$ respectively. No statistical significant difference observed in MPD diameter between the genders ( $p>0.05)$. (Table 2) 
To calculate age related changes of MPD sample was divided in to 2 groups of $<40$ and $>40$ years. Numbers of patients included in each group were 563 and 339 respectively. In $<40$ age group MPD diameter of pancreas in head, body and tail region were $2.9 \mathrm{~mm}, 2.2 \mathrm{~mm}, 1.6 \mathrm{~mm}$. Same measurements were obtained for $>40$ years age group. No statistical significant difference observed in MPD diameter between the two age groups of $<40$ and $>40$ years (p>0.05). (Table 3)

\section{Discussion}

Different imaging modalities such as USG, CT, MRCP, ERCP and PTC are used for the assessment of MPD in adults. Most of the previous studies which were done to study the pancreatic duct diameter were based on USG. Limitations concerning the USG are that poor resolution of most machines and the fact that measurements are affected by the technique ${ }^{6}$. Furthermore, for obese individuals, delineation of the pancreas with US might be challenging ${ }^{6}$. Although MRCP clearly depicts MPD dilatation, it is costly and not readily accessible in Sri Lanka. Despite being considered as gold standard for assessing the cause of obstructive jaundice, ERCP is invasive, thus less suitable in adults with pancreatic disorders ${ }^{6}$.

"CT has been used in clinical practice for more than two decades. Pancreatic duct width measurements in CT are now used as one of the major criteria for diagnosing certain pancreatic diseases, such as pancreatitis and pancreatic cancer." 8,9 Even though there are no approved recommendations to identify dilation of pancreatic duct, it is not hard to detect pancreatic duct dilation on CT.

Numerous research studies during 1980s showed that MPD could be revealed by ultrasound scans in 50 to $82 \%$ of persons. ${ }^{10,11}$ Over the last decade, sonography demonstrated 75 to $90 \%$ rate of sensitivity to detect the MPD diameter in adults ${ }^{12}$. In the current study the pancreatic duct was adequately visualized only in $72.9 \%$ of subjects in CT. Surprisingly this figure is comparatively less than the sensitivity of USG to determine the diameter of pancreatic duct, according to published data. Since available data on sensitivity values of CT are lacking, more research should be done on this.

We minimized the error of measurements of the pancreatic duct by obtaining the average of three measurements. Unlike USG on which most previous studies of MPD measurements were performed, CECT has the advantage of lack of operator dependence. We have further improved the accuracy of measurements by engaging two experience registrars in Radiology to obtain the measurements.

"The normal width of the pancreatic duct in a healthy adult is slightly different in the head, body and tail of the pancreas." 8,9 Our prospective study demonstrates that the mean diameter of the MPD in the head, body and tail were $2.9 \pm 0.4 \mathrm{~mm}, 2.2 \pm 0.3 \mathrm{~mm}$ and $1.6 \pm 0.2 \mathrm{~mm}$ respectively. The diameter values of MPD in our study were similar to foreign population groups studied such as in Asia, Europe and America by Hadidi A, Mortelé KJ and coworkers ${ }^{4}$. The most remarkable discovery of this study is that there is no statistical significant difference observed in MPD diameter between the two age groups of $<40$ and $>40$ years $(p>0.05)$ 
implying no correlation between MPD diameter with the age. However, this finding was different to the previous studies which showed higher proportion of MPD diameter with increasing age with significant rise from the fifth decade of life on words. Furthermore the literature describes, "although the width may be affected by age, variation should not be greater than $1 \mathrm{~mm}$ in each part of the pancreas"13.

According to our experience, no correlation was demonstrated between the main pancreatic duct diameter and the sex. But Glaser et al revealed that "the age-dependent caliber enlargement was more distinct in females than in males"7.

"Slight dilatation of the main pancreatic duct appears to be a risk factor for pancreatic cancer"6 "Thus, in such subjects without pancreatic cancer, periodic checkups with a non-invasive modality are recommended for the early detection of pancreatic cancer" 6 . Therefore, the reference values of the normal size of the MPD in Sri Lankan population published in the present study is invaluable for early detection of pancreatic pathologies.

In conclusion, CT measurement of main pancreatic duct diameter in our study is comparable to that of foreign population groups. However there was no correlation between MPD diameter with the age or sex.

\section{Limitations and future research}

Since this study recruited participants in Colombo South Teaching Hospital, Kalubowila, participants tended to be from a specific geographical location in Sri Lanka. Therefore, the current study was not truly a population-based study involving Sri Lanka, but a study at a tertiary care center.

For future research, diversifying the sampling frame and increasing sample size could result in a more accurate result. 


\section{Acknowledgments}

We would like to express our appreciation to Consultant Radiologist, Dr. Apsara Epa for her persistent guidance, encouragement and valuable critiques of this research work.

My deep gratitude also extended to Co Registrars for the support and encouragement throughout the study.

\section{References}

1. Mark D Edge, Maarouf Hoteit, Amil P Patel, Xiaoping Wang, Deborah A Baumgarten, Qiang Cai, "Clinical significance of main pancreatic duct dilation on computed tomography: Single and double duct dilation".World J Gastroenterol 2007 March 21; 13(11): 1701-1705

2. Tanaka S, Nakao M, Ioka T et-al. "Slight dilatation of the main pancreatic duct and presence of pancreatic cysts as predictive signs of pancreatic cancer: a prospective study". Radiology. 2010;254 (3): 965-72. Radiology (full text) - doi:10.1148/radiol.09090992 - Pubmed citation

3. Satoi, Shumpei; Takeyama, Yoshifumi; Nakai, Takuya et al. "Diameter of Main Pancreatic Duct Is Important for Prediction of Malignancy of IPMN”. Pancreas:July 2009 - Volume 38 Issue 5 - $\mathrm{p} 481$

4. Hadidi A." Pancreatic duct diameter: sonographic measurement in normal subjects". J Clin Ultrasound. 1983 Jan;11(1):17-22

5. Mortelé KJ, Rocha TC, Streeter JL et-al. "Multimodality imaging of pancreatic and biliary congenital anomalies". Radiographics. 2006;26 (3): 715-31. doi:10.1148/rg.263055164 Pubmed citation

6. Sachiko Tanaka, Miho Nakao et al. "Slight Dilatation of the Main Pancreatic Duct and Presence of Pancreatic Cysts as Predictive Signs of Pancreatic Cancer: A Prospective Study". RSNA RADIOLOGY March 2010:Volume 254, Issue 3

7. J. Glaser, B. Hogemann, T. Krummenerl, M. Schneider, E. Hultsch, N. Van Husen, and U. Gerlach. "Sonographic Imaging of the Pancreatic Duct". Digestive Diseases and Sciences, Vol. 32, No. 10 (October 1987), pp. 1075-1081

8. Sullivan LM. In The liver, biliary system and the Pancreas.In: Juhl JH, Crummy AB, Kuhlman JE. Essentials of Radiology Images. 7th ed. Philadelphia: Lippincott Williams \& Wilkins, 1998: 511-544

9. Haaga JR. The pancreas. In: Haana JR. Computed Tomography \& Magnetic Resonance Image of the Whole Body. 3rd ed. St.Louis: Mosby, 1994: 1035-1130

10. Weinstein DP, Weinstein BJ: Ultrasonic demonstration of the pancreatic duct: An analysis of 41 cases. Radiology 130:729, 1979

11. Parulekar SG: Ultrasonic evaluation of the pancreatic duct. J Clin Ultrasound 8:457, 1980

12. H.Chin Chao, SJ Lin, MS Kong and CC Luo" Sonographic evaluation of the pancreatic duct in normal children and children with pancreatitis" J Ultrasound Med 19:757-763,2000 
13. Das SD, Tassios PS, Giorgiotis K, Rokkas T, Theodosiou P, Raptis SA. Pancreatic duct width: its significance as a diagnostic criterion for pancreatic disease. Hepatogastroenterology 1993; 40:52-55

Table 1: MPD size measurements according to the region of MPD

\begin{tabular}{|l|r|l|l|}
\hline Region of MPD & $\begin{array}{l}\text { Mean } \\
\text { value }(\mathrm{mm})\end{array}$ & $\begin{array}{l}\text { Maximum } \\
\text { value(mm) }\end{array}$ & $\begin{array}{l}\text { Minimum } \\
\text { value(mm) }\end{array}$ \\
\hline Head & $2.9 \pm 0.4$ & 4.5 & 1.8 \\
\hline Body & $2.2 \pm 0.3$ & 3.4 & 1.5 \\
\hline Tail & $1.6 \pm 0.2$ & 3.4 & 1.5 \\
\hline
\end{tabular}

Table 2: Statistical analysis of MPD diameter between genders according to the region of MPD

\begin{tabular}{|l|l|}
\hline Region of MPD & $\begin{array}{l}\text { P } \\
\text { Value }\end{array}$ \\
\hline Head & 0.62 \\
\hline Body & 0.34 \\
\hline Tail & 0.74 \\
\hline
\end{tabular}

Statistical method: chi-square test.

Table 3: Statistical analysis of MPD diameter between two age groups $(<40)$ and $>40 y e a r s$

\begin{tabular}{|c|c|}
\hline Region of MPD & $\begin{array}{l}\mathrm{P} \\
\text { Value }\end{array}$ \\
\hline Head & 0.93 \\
\hline Body & 0.92 \\
\hline Tail & 0.20 \\
\hline
\end{tabular}

Statistical method: chi-square test. 
Figure 1: Participant's characteristics- sex

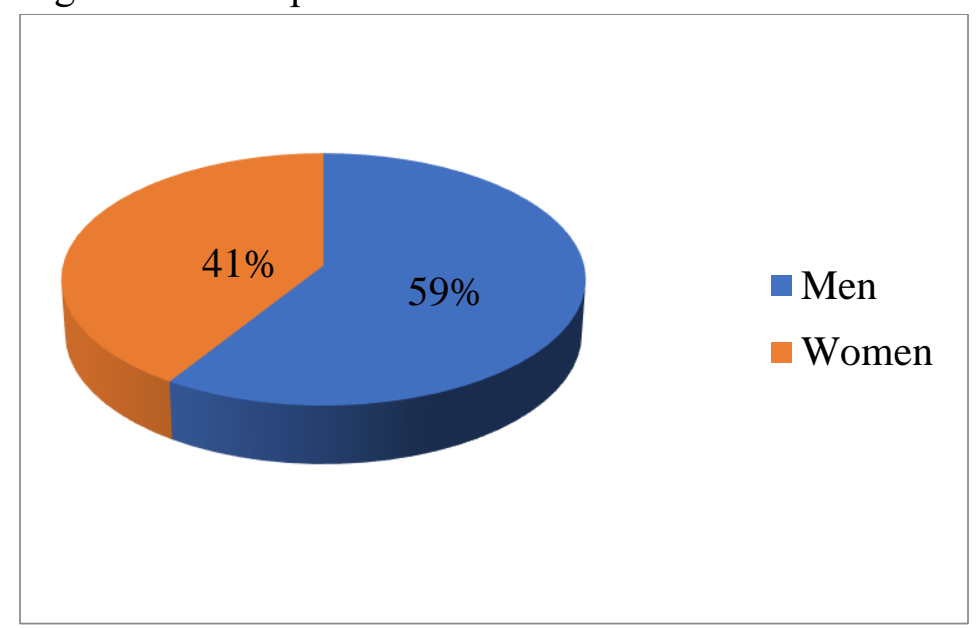

Figure 2: Participant's characteristics- Age

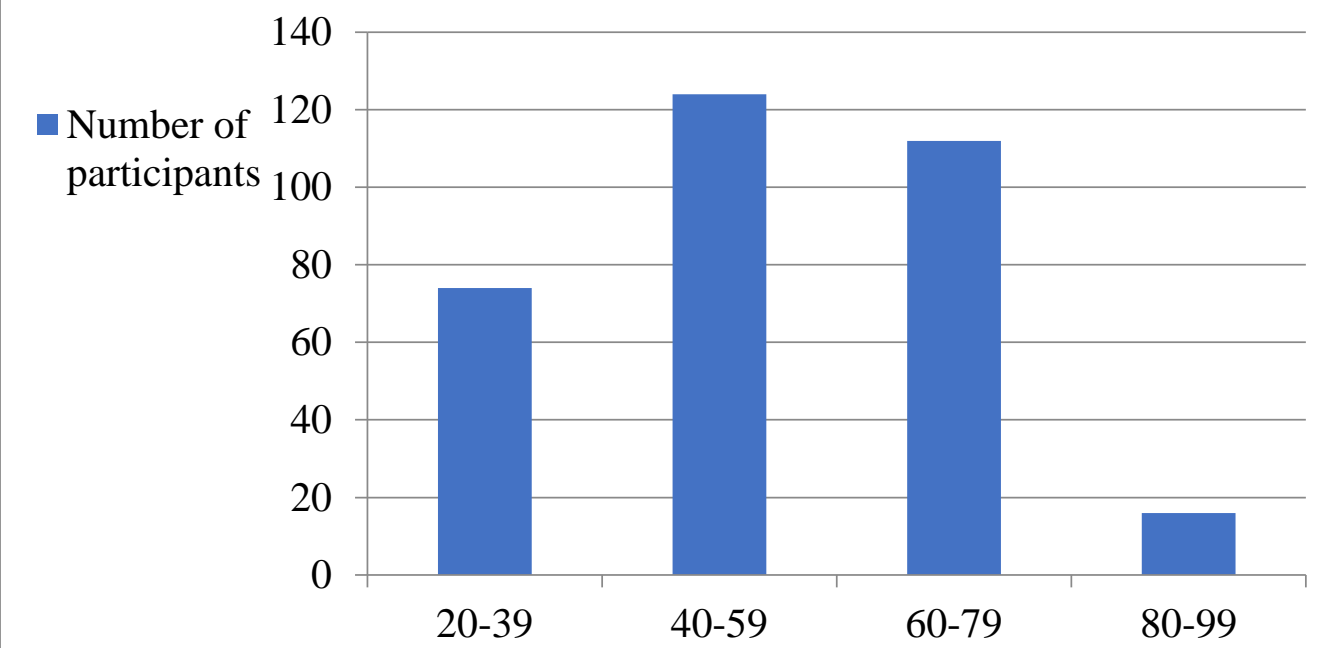

Figure 3: Adequate visualization of MPD

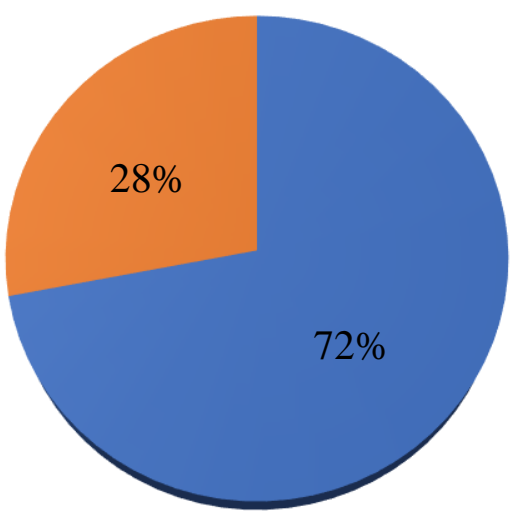

- Adequately visualised

Adequately not visualised 